\title{
Polytropic Behavior of Solar Wind Protons Observed by Parker Solar Probe
}

\author{
Georgios Nicolaou $^{1}$, George Livadiotis ${ }^{2}$ (1) , Robert T. Wicks ${ }^{1,3}$ (1) , Daniel Verscharen ${ }^{1,4}$ (i), and Bennett A. Maruca ${ }^{5}$ (1) \\ ${ }^{1}$ Department of Space and Climate Physics, Mullard Space Science Laboratory, University College London, Dorking, Surrey, RH5 6NT, UK; g.nicolaou@ucl.ac.uk \\ ${ }^{2}$ Division of Space Science and Engineering, Southwest Research Institute, San Antonio, TX 78238, USA \\ ${ }^{3}$ Department of Mathematics, Physics and Electrical Engineering, Northumbria University, Newcastle-upon-Tyne, NE1 8ST, UK \\ ${ }^{4}$ Space Science Center and Department of Physics, University of New Hampshire, Durham, NH 03824, USA \\ ${ }^{5}$ Bartol Research Institute, Department of Physics and Astronomy, University of Delaware, Newark, DE 19716, USA \\ Received 2020 June 9; revised 2020 July 21; accepted 2020 July 28; published 2020 September 17
}

\begin{abstract}
A polytropic process describes the transition of a fluid from one state to another through a specific relationship between the fluid density and temperature. The value of the polytropic index that governs this relationship determines the heat transfer and the effective degrees of freedom during a specific process. In this study, we analyze solar wind proton plasma measurements, obtained by the Faraday cup instrument on board the Parker Solar Probe. We examine the large-scale variations of the proton plasma density and temperature within the inner heliosphere explored by the spacecraft. We then address the polytropic behavior in the density and temperature fluctuations in short time intervals, which we analyze in order to derive the effective polytropic index of smallscale processes. The large-scale variations of the solar wind proton density and temperature, which are associated with the plasma expansion into the heliosphere, follow a polytropic model with a polytropic index $\sim 5 / 3$. On the other hand, the short-scale fluctuations, which are potentially associated with turbulence, follow a model with a larger polytropic index. We investigate possible correlations between the polytropic index of short-scale fluctuations and the plasma speed, plasma $\beta$, and the magnetic field direction. We discuss candidate mechanisms leading to this behavior including energy transfer and possible mechanisms restricting the effective particle degrees of freedom at smaller scales.
\end{abstract}

Unified Astronomy Thesaurus concepts: Solar wind (1534); Space plasmas (1544); Solar physics (1476)

\section{Introduction}

A polytropic process is any thermodynamic transition in which the density $n$ and temperature $T$ (or pressure $P$ ) of a fluid that obeys the ideal gas law are related through the polytropic index $\gamma$ through

$$
T \propto n^{\gamma-1} \text { or } P \propto n^{\gamma} .
$$

During a polytropic process, the ratio between the energy transferred into the system as heat and the energy transferred as work remains constant (e.g., Parker 1963; Chandrasekhar 1967). In the special case when there is no heat transfer in the system during the transition, the process is called adiabatic. In an adiabatic process, $\gamma$ is equal to the ratio of the specific heat under constant pressure over the specific heat under constant volume $c_{\mathrm{p}} / c_{\mathrm{v}}$, and is related with the degrees of freedom $f$ of the plasma particles, by $\gamma=c_{\mathrm{p}} / c_{\mathrm{v}}=1+\frac{2}{f}$. The knowledge of $\gamma$ is essential for the fluid (including MHD) description of plasma processes and thus, necessary for the understanding of several physical processes in plasma environments. As we cannot directly measure the value of $\gamma$ in space, we validate models of the polytropic behavior in order to understand the relevant physics processes in comparison with observations. The polytropic equation brings closure to the moment hierarchy by connecting higher-order moments $(T, P)$ with the zerothorder moment $(n)$ of the velocity distribution function of plasma particles (e.g., Kuhn et al. 2010). Moreover, through the polytropic equation, we approximate the physics of transitions in the medium without the need to solve the explicit energy equation describing the system. For instance, $\gamma$ defines the compression ratio of shocks (e.g., Parker 1961; Livadiotis 2015; Scherer et al. 2016; Nicolaou \& Livadiotis 2017), the nature of turbulent fluctuations and waves (e.g., Bavassano et al. 1996;
Verscharen et al. 2016, 2017, 2019; Wu et al. 2019), the expansion of plasma within several environments, such as planetary magnetospheres (e.g., Spreiter \& Stahara 1994), the interplanetary space (e.g., Elliott et al. 2019), and the heliosheath (e.g., Livadiotis et al. 2011; Livadiotis \& McComas 2013). Importantly, recent studies argue that the fluid approach describes successfully small-scale fluctuations, even in plasmas with low collisionality, such as the plasma protons in the solar wind and the terrestrial magnetosheath (e.g., Verscharen et al. 2017, 2019; Wu et al. 2019). Therefore, the polytropic description is possibly applicable within a wide range of plasma scales.

The value of $\gamma$ characterizes specific processes in individual fluid parcels and, thus, may be different within different plasma regimes, for different plasma species, and/or it may vary with time. Moreover, Livadiotis (2016) analyzes streams that consist of multiple polytropes. Some studies investigate the polytropic behavior of plasmas in large-scale processes, such as the solar wind expansion within the heliosphere. For instance, Totten et al. (1995) determine $\gamma$ through the radial profiles of the solar wind proton density and temperature within a wide range of heliocentric distances. The derived $\gamma$ leads to the conclusion that the solar wind plasma protons are heated as they propagate in the outer heliosphere.

In other representative analyses, $\gamma$ is determined in individual streams where Equation (1) is valid. For example, studies determine the polytropic behavior in streams of space plasma species within several regimes, such us planetary magnetospheres (e.g., Arridge et al. 2009; Nicolaou et al. 2014b; Pang et al. 2015; Dialynas et al. 2018; Park et al. 2019), magnetic clouds (e.g., Osherovich et al. 1993), the solar wind (e.g., Newbury et al. 1997; Kartalev et al. 2006; Nicolaou et al. 2014a, 2019; 

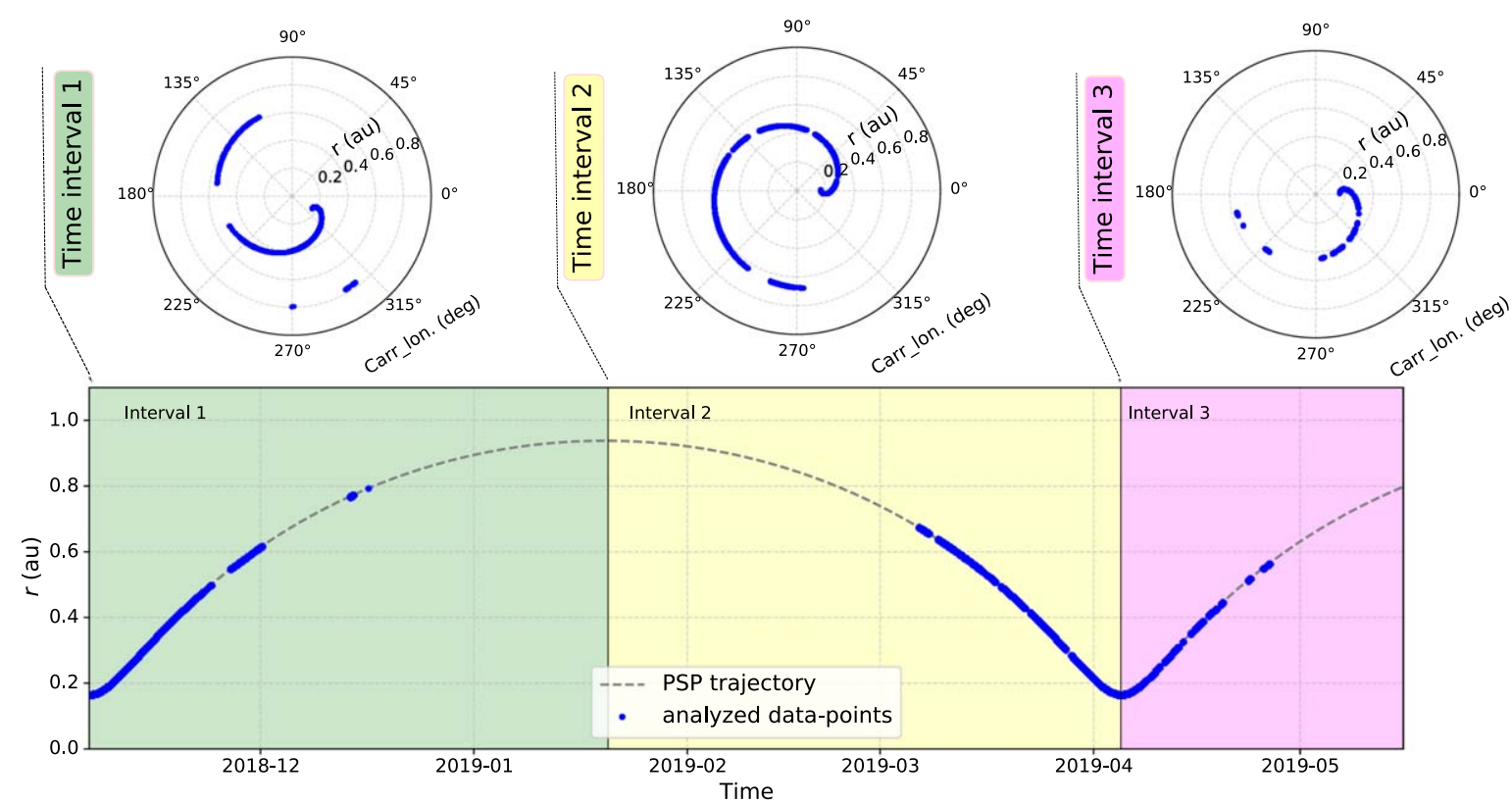

Figure 1. Heliocentric distance of PSP from 2018 November 6 to 2019 May 15. The shadowed regions correspond to the three time intervals we analyze separately in this study. The blue data points correspond to the observations that are flagged as "measurements with no conditions" and small relative density and temperature uncertainties $\left(\sigma_{n} / n<30 \%\right.$ and $\left.\sigma_{T} / T<30 \%\right)$, which we analyze in this study. The panels at the top show the spacecraft longitude in the Carrington coordinate system for the three time intervals.

Livadiotis \& Desai 2016; Livadiotis 2018a, 2018b; Elliott et al. 2019; Nicolaou \& Livadiotis 2019), and the inner heliosheath (e.g., Livadiotis et al. 2013).

In this study, we analyze observations by NASA's Parker Solar Probe (PSP). We use data obtained within distances of $\sim 0.17$ and $\sim 0.8$ au $\left(1\right.$ au $\left.\sim 1.5 \times 10^{8} \mathrm{~km}\right)$ from the Sun, giving us the opportunity to investigate the macroscopic changes of the plasma parameters as the solar wind expands into the heliosphere. The high-time-resolution measurements of solar wind protons also allow the investigation of the polytropic indices on very small scales, associated with plasma compressive fluctuations due to waves and turbulence. We also investigate if the polytropic behavior of the plasma protons depends on the solar wind speed $U$, which is often used as the basic criterion to separate between solar wind plasmas of different solar origins (e.g., McComas et al. 2003) and with different properties (e.g., Marsch et al. 1982, 1983; Geiss et al. 1995; Hellinger et al. 2011; Borovsky 2016; Perrone et al. 2019; Stansby et al. 2019; Huang et al. 2020). Finally, we examine the polytropic index as a function of the plasma $\beta$, and the magnetic field direction. In the next section we describe the data set we use in this study. In Section 3, we describe our methods and Section 4 shows our results, which we discuss in Section 5. Finally, in Section 6, we summarize our conclusions.

\section{Data}

Solar Probe Cup (SPC), part of the Solar Wind Electrons Alphas and Protons (SWEAP) suite (Kasper et al. 2016), is a Sun-pointing Faraday cup capturing the radial bulk flow of the plasma particles. The flux, integrated over the field of view $\left(\sim 60^{\circ}\right)$, is converted to 1D distribution functions and analyzed to derive the plasma bulk properties. Here, we use the plasma proton density, temperature, and speed as derived from fits to the spectra observed by SPC. We use the plasma observations obtained between 2018 November 6 and 2019 May 16. The specific time interval includes two encounters to the Sun at $\sim 0.17 \mathrm{au}$. We separate the selected data set into three consecutive time intervals, in a way that each interval covers the maximum possible heliocentric distance range (see the bottom panel of Figure 1). Large ranges in heliocentric distance correspond to, on average, large variations in the plasma density and temperature allowing the investigation of the polytropic relation within the inner heliosphere. During each interval, the spacecraft samples streams over a wide range of Carrington longitudes (top panel of Figure 1). Interval 1 starts at PSP's perihelion on 2018 November 6 and ends at the aphelion on 2019 January 20, when the second interval begins. Interval 2 ends at the perihelion on 2019 April 4, when the third interval begins. Interval 3 ends on 2019 May 15.

Each set of SPC Level 3 proton parameter values carries standardized quality flags that can indicate various issues with the data: e.g., poor sampling, spacecraft maneuvers, or poor convergence of the fitting algorithm. Per the recommendation of the "User Guide for Parker Solar Probe SWEAP Investigation Data Products ${ }^{6}$," we only consider the general flag, which is a composite of the other flags and gives an overall indication of whether a datum is suitable for science. We also select data points with relative density and temperature uncertainty $\sigma_{n} / n<30 \%$ and $\sigma_{T} / T<30 \%$, respectively, where $\sigma$ denotes the $1 \sigma$ uncertainty of the derived parameters. All of these criteria were met by $\sim 29 \%$ of the data obtained within the total time period we examine. For our investigation of the polytropic behavior dependence on the plasma $\beta$ and the magnetic field direction which potentially affects the derived temperature (see Section 4.6), we use a 2 day time interval, between 2019 April 2 and 2019 April 4. For the study of this time interval, we use high-time-resolution magnetic field observations from the Electromagnetic Fields Investigation

\footnotetext{
6 http://sweap.cfa.harvard.edu/Data.html
} 

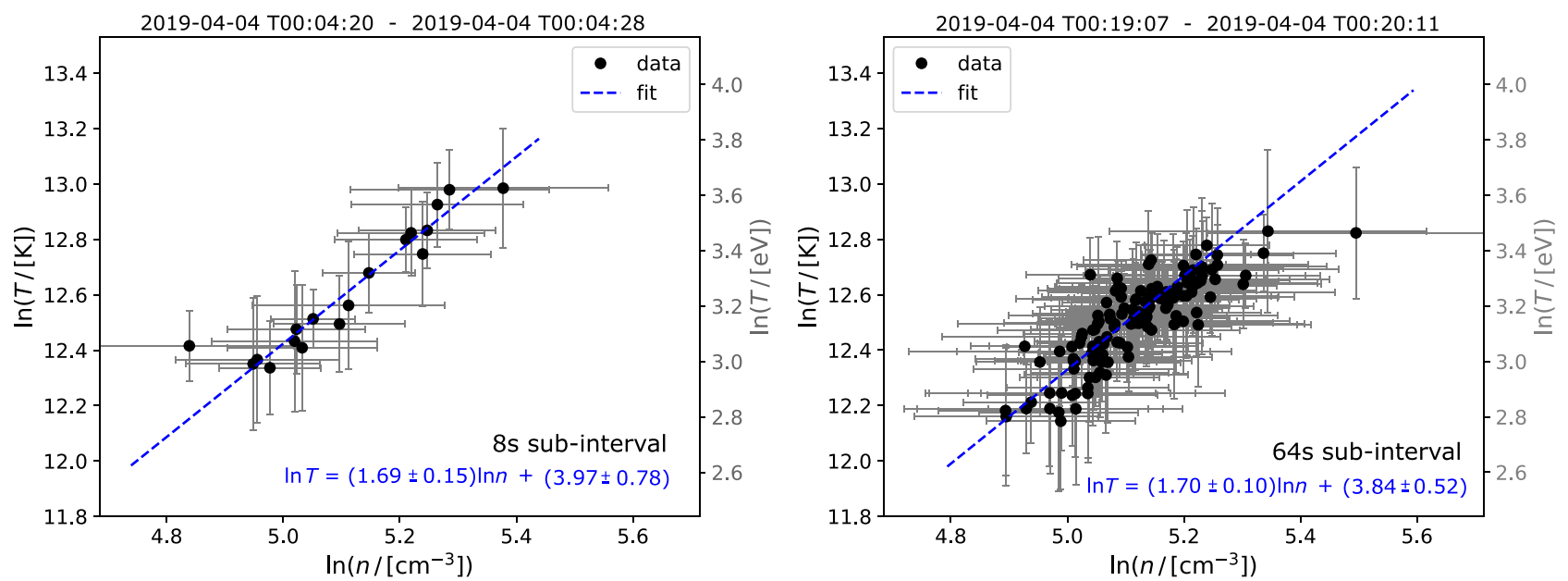

Figure 2. $\ln T$ as a function of $\ln n$ within a typical subinterval of (left) $8 \mathrm{~s}$ and (right) $64 \mathrm{~s}$. The vertical (horizontal) error bars correspond to $\sigma_{T} / T\left(\sigma_{n} / n\right.$ ). In both panels, the blue line is the linear fit result of Equation (2) to the data points. The slope of the fitted line determines $\gamma$. In both examples, the slope of the fit result is $\sim 1.7$, which corresponds to $\gamma \sim 2.7$.

(FIELDS; Bale et al. 2016) instrument, obtained within the specific interval.

\section{Methodology}

According to Equation (1):

$$
\log _{10}(T)=(\gamma-1) \log _{10}(n)+C,
$$

where the constant $C$ is generally different for different plasma streams. We initially examine the two-dimensional (2D) histograms of $\log _{10}(n)$ and $\log _{10}(T)$ in order to investigate whether the large-scale plasma expansion in the heliosphere follows the polytropic model in Equation (2). As different streams cross the spacecraft during the examined intervals, the linear relationship in Equation (2) can be determined only if the change of $C$ across the different streams is smaller than the large-scale variations of $\log _{10}(n)$ and $\log _{10}(T)$. Therefore, we do not attempt to determine a unique polytropic relation using the large-scale variations of the plasma. Instead, we calculate $\gamma$ and its $1 \sigma$ error $\sigma_{\gamma}$ on short timescales by dividing each interval into smaller consecutive subintervals and performing a weighted orthogonal-distance regression on $\ln T$ versus $\ln n$ within each subinterval. We use weighted orthogonal regression, considering symmetric uncertainty $\sigma_{\ln T} \sim \sigma_{T} / T$ and $\sigma_{\ln n} \sim \sigma_{n} / n$ for each $\ln T$ and $\ln n$ data point respectively. We specifically examine subintervals of 8,64 , and $512 \mathrm{~s}$. The plasma fluctuations on such short timescales are mainly due to transient compressions and compressive fluctuations associated with waves and other local plasma fluctuations. To increase the statistical significance of the results, we reject subintervals with fewer than five data points and those for which $\sigma_{\gamma}>1$. The short length of the examined subintervals eliminates the possibility to mix observations of different streams (e.g., Kartalev et al. 2006; Nicolaou et al. 2014a, 2019; Pang et al. 2015; Nicolaou \& Livadiotis 2019). In Figure 2, we show an example of $\ln T$ as a function of $\ln n$ within a typical $8 \mathrm{~s}$ subinterval (left) and a typical $64 \mathrm{~s}$ subinterval (right). The data points within the shown subintervals follow a clear linear behavior described by the fit of our model in Equation (2) to the observations. The slope of the fit in each subinterval determines $\gamma$. The fits to the subintervals in Figure 2 have the same slope $(\sim 1.7)$, which corresponds to $\gamma \sim 2.7$. The two lines, however, have a different $\ln (T)$-intersect, corresponding to a different $C$ constant in each subinterval.

\section{Results \\ 4.1. Histograms of Large-scale Variations}

In Figure 3, we show 2D histograms of $\log _{10}(n)$ and $\log _{10}(T)$ for the three time intervals shown in Figure 1. In order to enhance features with low occurrence, we normalize each histogram column to its maximum occurrence value. In each panel, we overplot adiabatic models for protons with three degrees of freedom ( $f=3, \gamma=5 / 3)$ as guides to the eye. As mentioned in Section 3, different plasma streams pass the spacecraft within the sampled heliocentric distance range making it impossible to determine a single polytropic relationship describing the whole data set within each interval. On the other hand, we investigate whether the statistical sampling of streams within the examined heliocentric distance range reveals a characteristic slope, and hence polytropic behavior that describes the large-scale variations of $\log _{10}(n)$ and $\log _{10}(T)$ associated with the expanding solar wind in the heliosphere. Within each interval we examine here, the proton density ranges from $\sim 10$ to $\sim 1000 \mathrm{~cm}^{-3}$, while the proton temperature ranges from $\sim 3 \times 10^{4}$ to $\sim 3 \times 10^{6} \mathrm{~K}$. The large-scale variations of $n$ and $T$ within all three intervals tend to follow the adiabatic model, especially when $n<100 \mathrm{~cm}^{-3}$. Within the higher density regime $\left(n>100 \mathrm{~cm}^{-3}\right)$, the temperature drops below the adiabatic model, especially in intervals 1 and 3 , while in interval 2 we still observe high occurrence along the adiabatic line. Moreover, within intervals 1 and 2, we observe discrete bright structures that deviate from the $\gamma=5 / 3$ behavior as their slopes are steeper than the slope of the adiabatic model.

\subsection{Density and Temperature versus Radial Distance}

As the solar wind expands in the heliosphere, its density decreases. For a polytropic expansion, the plasma temperature will change accordingly. In the top panel of Figure 4, we show the $2 \mathrm{D}$ histogram of the proton number density and the radial 

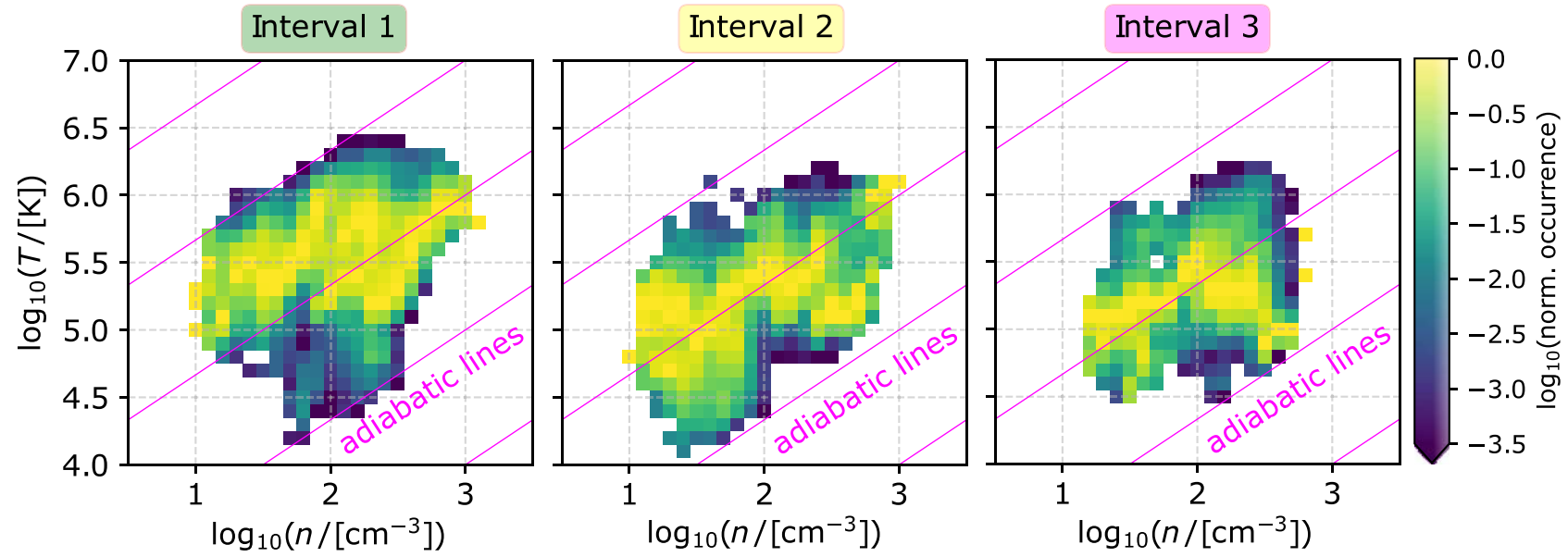

Figure 3. Two-dimensional histograms of $\log _{10}(n)$ and $\log _{10}(T)$ for the three time intervals shown in Figure 1. In each panel, we show adiabatic lines for protons with three degrees of freedom $(f=3, \gamma=5 / 3)$. Typically, the large-scale changes of the plasma density and temperature within each subinterval follow a near-adiabatic trend. However, we observe features with $\gamma>5 / 3$.

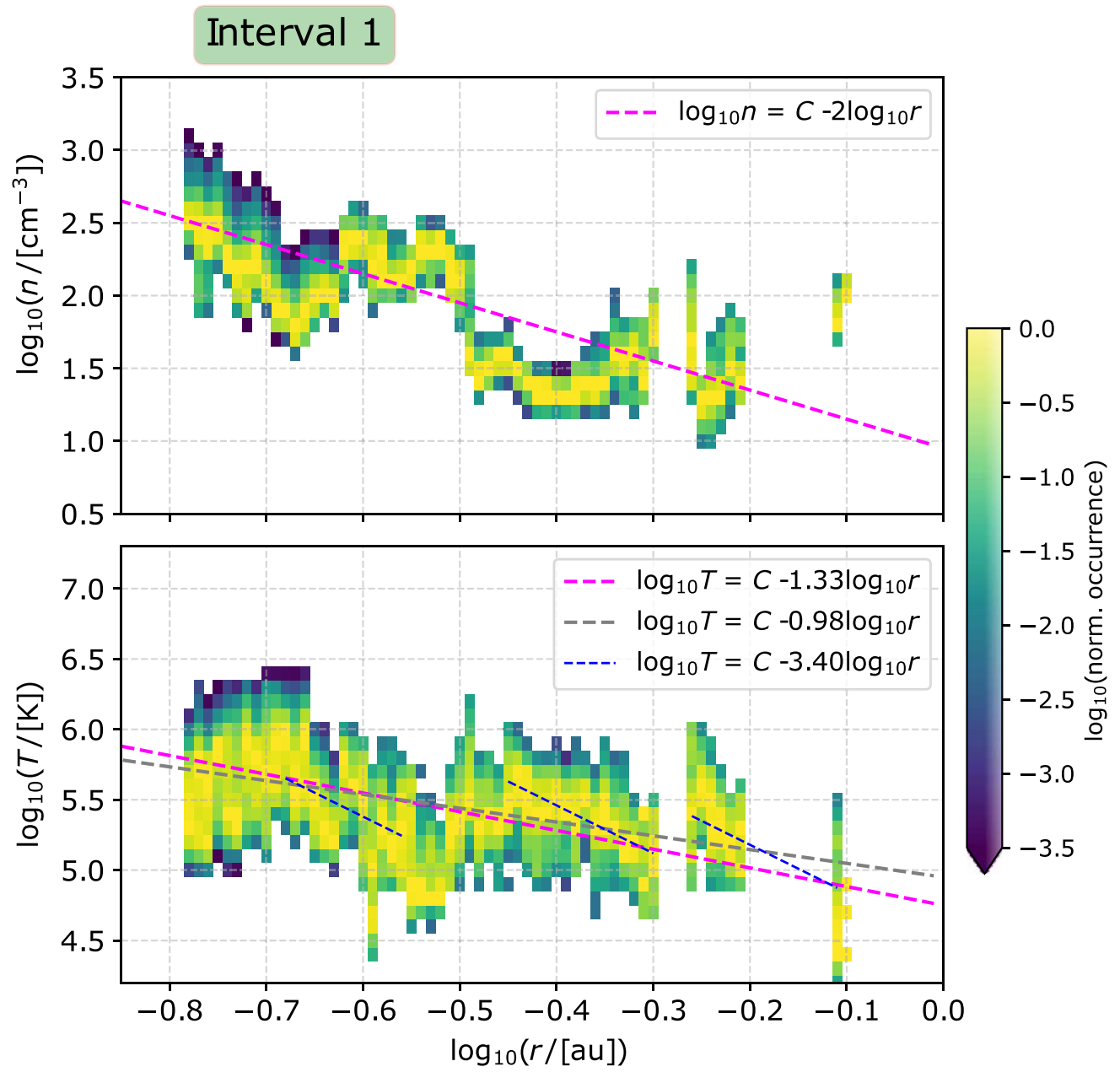

Figure 4. Two-dimensional histograms of (top) the proton density and (bottom) the proton temperature as functions of the radial distance for time interval 1 . The magenta line in the top panel shows the expected density for an expansion model with constant speed, $n \propto r^{-2}$. In the lower panel, the magenta line shows the expected temperature of a polytropic radial expansion model with $\gamma=5 / 3$ while the blue lines represent expansion models with $\gamma=2.7$. The gray line illustrates the slope determined by Huang et al. (2020) for the parallel proton temperature of fast solar wind observed by SPC.

distance for interval 1 . The magenta line shows the model for uniform expansion with constant radial velocity, $n \propto r^{-2}$. Although $n \propto r^{-2}$ captures basic features of the entire profile, we distinguish regions where the decrement of $n$ with $r$ is either steeper or flatter. The bottom panel of Figure 4 shows the 2D histogram of temperature and the radial distance for the same interval. The magenta line shows an expansion model with $\gamma=5 / 3$, assuming a constant radial speed, so that $n \propto r^{-2}$. 

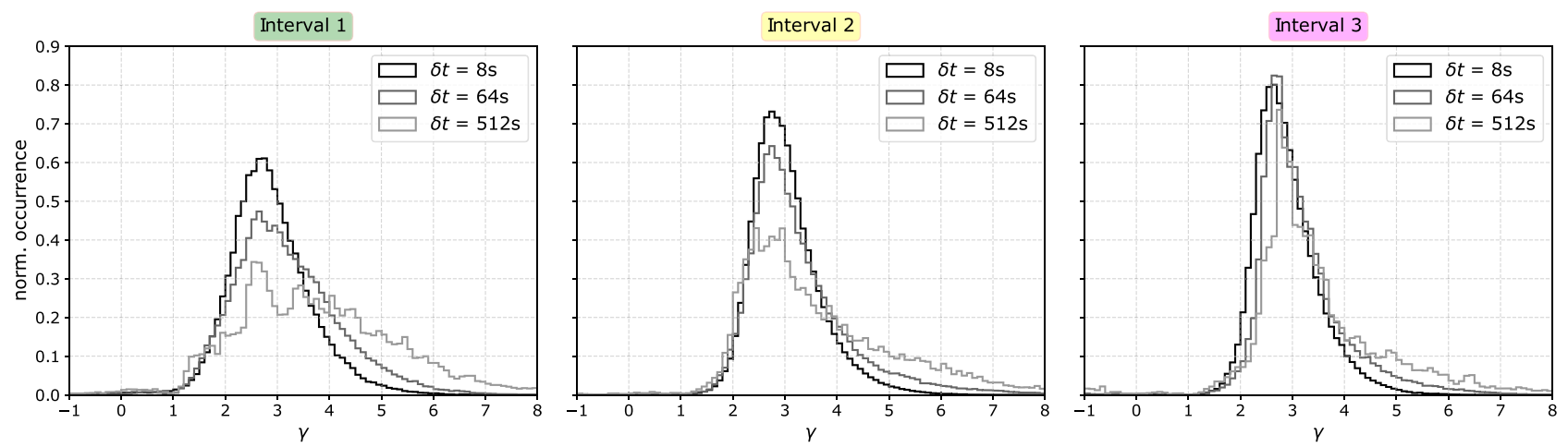

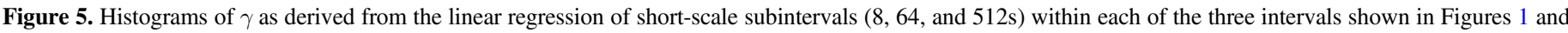
3 . The most frequent value is $\gamma \sim 2.7$, for every timescale we examine, and $\sigma_{\gamma}$ increases with increasing length $\delta t$ of the analyzed subintervals.

The gray line shows a radial profile with the same slope as the one characterizing the parallel proton temperature of fast solar wind protons determined by Huang et al. (2020) from the analysis of SPC data. Both of these models capture the largescale features of the entire profile. However, there are distinct subintervals with steeper slopes which are better described by the blue lines showing radial expansion models with $\gamma=2.7$. As we show in the next section, this is a typical value within short subintervals of the analyzed data set. We discuss further our results and their possible implications in Section 5.

\subsection{Small-scale Regression}

For a conclusive study of the polytropic behavior of the solar wind protons, we analyze short time intervals. The use of these short time intervals also eliminates the effects due to spacecraft crossings of multiple solar-wind streams. We analyze subintervals with a clear polytropic behavior by applying the selection criteria explained in Section 3. In Figure 5, we show histograms of $\gamma$, as derived in subintervals of 8,64 , and $512 \mathrm{~s}$ width within each of the time intervals 1,2 , and 3 . In Table 1, we show the most frequent values and standard deviations of the histograms in each interval. The most frequent value is $\gamma \sim 2.7$ for the majority of the examined histograms, while the standard deviation progressively increases with increasing length of the subintervals. Within interval 1, the standard deviation of the polytropic index is $\sigma_{\gamma} \sim 0.80$ for the $8 \mathrm{~s}$ subintervals, $\sigma_{\gamma} \sim 1.05$ for the $64 \mathrm{~s}$ subintervals, and $\sigma_{\gamma} \sim$ 1.80 for the $512 \mathrm{~s}$ subintervals. In interval $2, \sigma_{\gamma} \sim 0.67$ for the $8 \mathrm{~s}$ subintervals, which then increases to $\sigma_{\gamma} \sim 1.85$ for the $512 \mathrm{~s}$ subintervals. Finally, in interval 3 , the analysis of the $8 \mathrm{~s}$ subintervals leads to $\sigma_{\gamma} \sim 0.64$, which increases to $\sigma_{\gamma} \sim 1.90$ for the $512 \mathrm{~s}$ subintervals.

The linear fit of Equation (2) to the $\ln T-\ln n$ data points of different streams fails to accurately derive the index $\gamma$, if the mixed streams have either different $\gamma$, or/and a different constant $C$. Such complications are possibly responsible for the asymmetry of the histograms in Figure 5. In addition, we remind the reader that each interval covers different heliocentric distance and longitude ranges (see Figure 1). As a result, each interval may contain samples of plasma streams originating from solar source regions with very different characteristics, which could be the reason for the different characteristics of their corresponding histograms. Since the possibility of stream mixing is reduced for the shortest subinterval length, hereafter, we use the $8 \mathrm{~s}$ subinterval results
Table 1

The Most Frequent Values and Standard Deviations of $\gamma$ in the Histograms Shown in Figure 4

\begin{tabular}{lccc}
\hline \hline $\begin{array}{l}\text { Interval } \\
\text { Name }\end{array}$ & $\begin{array}{c}\text { Subinterval } \\
\text { Duration } \delta t(\mathrm{~s})\end{array}$ & $\begin{array}{c}\text { Most Frequent } \gamma \\
\text { Value }\end{array}$ & $\begin{array}{c}\text { Standard } \\
\text { Deviation } \sigma_{\gamma}\end{array}$ \\
\hline Interval 1 & 8 & 2.7 & 0.8 \\
& 64 & 2.7 & 1.1 \\
Interval 2 & 512 & 2.6 & 1.8 \\
& 8 & 2.7 & 0.7 \\
& 64 & 2.7 & 1.1 \\
Interval 3 & 512 & 2.5 & 1.9 \\
& 8 & 2.7 & 0.6 \\
& 64 & 2.7 & 1.0 \\
& 512 & 2.8 & 1.9 \\
\hline
\end{tabular}

to explore further the polytropic behavior of the solar wind protons.

\subsection{Polytropic Behavior versus Speed}

We examine the polytropic behavior of the solar wind depending on its speed in an attempt to investigate whether plasma of different origins follows different polytropic scalings. In the left panel of Figure 6, we show the 2D histogram of $\gamma$ as derived from the analysis of $8 \mathrm{~s}$ subintervals in interval 1 and the average $U$ within the corresponding subintervals. On the right and top sides of the panel, we show the 1D histograms of $\gamma$ and $U$, respectively. The right panel of Figure 6 shows the 2D histogram of $\gamma$ and $U$ normalized to its maximum occurrence in each $U$ bin. The magenta lines in both panels show the most frequent value of $\gamma$ as a function of $U$. The speed within this interval ranges from $\sim 250$ to $\sim 700 \mathrm{kms}^{-1}$. The most frequent $\gamma \sim 2.7$ does not exhibit any systematic variations with speed.

\subsection{Polytropic Behavior versus Plasma $\beta$}

The proton plasma $-\beta=2 \mu_{0} n k T / B^{2}$ quantifies the partitioning of energy density between the plasma pressure ( $P=n k_{\mathrm{B}} T$, with $k_{\mathrm{B}}$ being the Boltzmann constant) and the magnetic field pressure $\left(P_{\mathrm{B}}=B^{2} /\left(2 \mu_{0}\right)\right.$, where $\mu_{0}$ is the vacuum permeability). Since we investigate thermodynamic processes in a magnetized plasma, it is important to explore the role of the plasma $\beta$, which plays a vital role in the polarization of plasma fluctuations (e.g., Bruno \& Carbone 2013; Chen et al. 2014; Franci et al. 2016; Verscharen et al. 2019; 


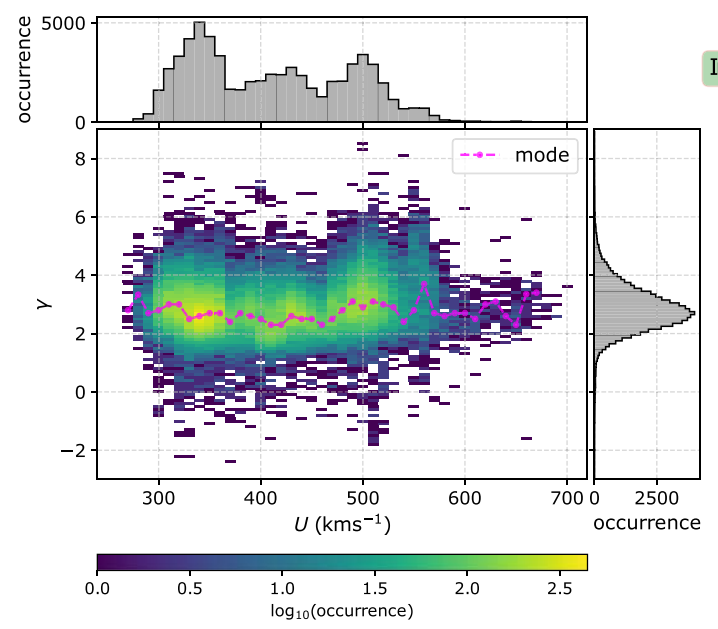

Interval 1

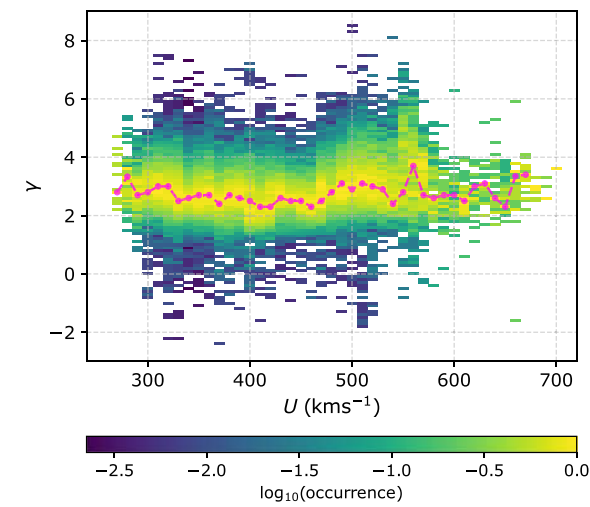

Figure 6. (Left) Two-dimensional histogram of $\gamma$ and $U$ in interval 1. On the right and top of the panel we show the 1D histograms of $\gamma$ and $U$ respectively. (Right) The 2D histogram of $\gamma$ and $U$, normalized to the maximum value per $U$ bin. The magenta lines in both panels show the most frequent value of $\gamma$ in each $U$ bin.
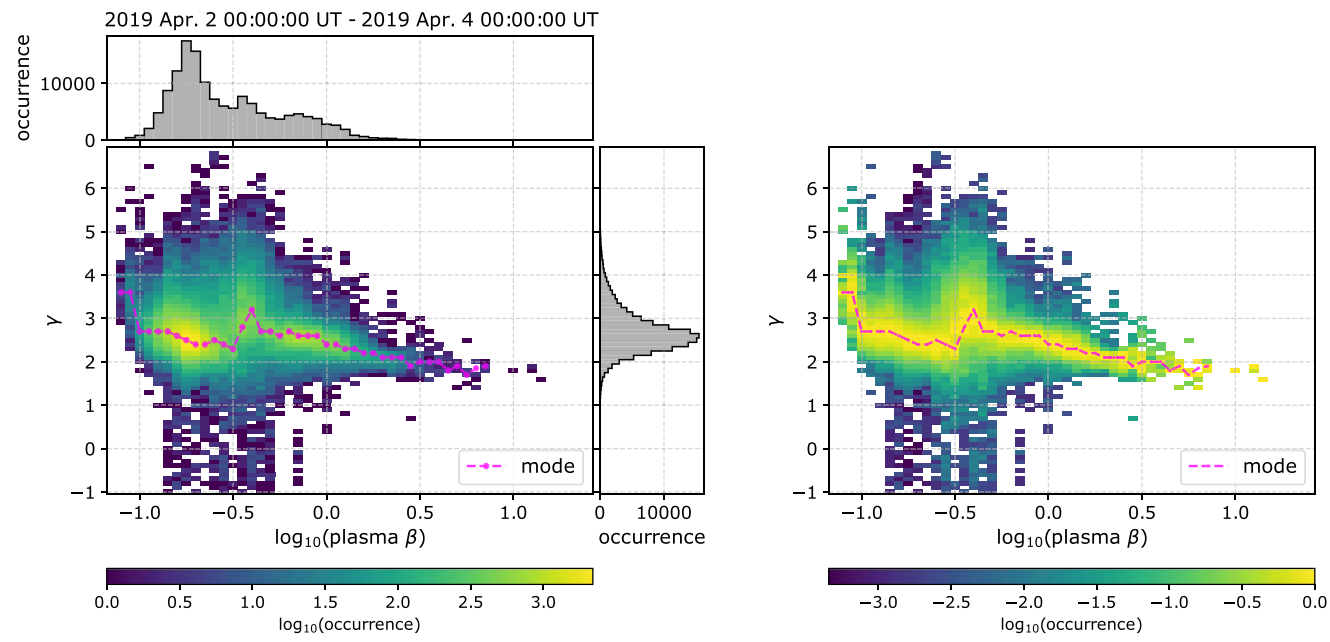

Figure 7. (Left) Two-dimensional histogram of $\gamma$ and $\log _{10}(\beta)$ for the time interval between 2019 April 2 00:00:00UT and 2019 April 4 00:00:00UT. On the right and top side of the panel, we show the $1 \mathrm{D}$ histograms of $\gamma$ and $\log _{10}(\beta)$ respectively. (Right) The $2 \mathrm{D}$ histogram of $\gamma$ and $\log _{10}(\beta)$, normalized to the maximum value per $\log _{10}(\beta)$ bin. In both panels, the magenta line shows the most frequent value of $\gamma$ in each $\log _{10}(\beta)$ bin.

Woodham et al. 2019). In the left panel of Figure 7, we show the $2 \mathrm{D}$ histogram of $\gamma$ and $\log _{10}(\beta)$ derived from our analysis of 8 s subintervals between 2019 April 2 and 2019 April 4. For this specific case study, we calculate $\beta$ from the average $n, T$, and $B$ over each $8 \mathrm{~s}$ subinterval to match the time resolution of the derived $\gamma$. On the right and top sides of the 2D histogram, we show the 1D histogram of $\gamma$ and the 1D histogram of $\log _{10}(\beta)$ for the same time period, respectively. The right panel of Figure 7 shows the 2D histogram of $\gamma$ and $\log _{10}(\beta)$ normalized to the maximum value in each $\log _{10}(\beta)$ bin. In both panels, the magenta line shows the most frequent value of $\gamma$ as a function of $\log _{10}(\beta)$. During the analyzed time period, $\log _{10}(\beta)$ ranges from -1.1 to 1 . We observe two distinct regions in the 2D histograms, where $\gamma$ decreases with $\beta$. More specifically, $\gamma$ decreases from $\sim 3.2$ to $\sim 2.5$ as $\log _{10}(\beta)$ increases from -1.1 to -0.5 . At $\log _{10}(\beta)-0.5$, there is a sharp increase of $\gamma$ from 2.5 to $\sim 3$, which is potentially associated with a crossing between different plasma streams. Finally, $\gamma$ decreases from 3 to $\sim 1.8$ as $\log _{10}(\beta)$ increases from -0.4 to 1 .

\subsection{Polytropic Behavior and B-field Orientation}

The solar wind protons often exhibit large temperature anisotropies, which are organized by the magnetic field direction (e.g., Marsch 2006; Verscharen et al. 2019, and references therein). There is also evidence that the evolution of turbulence is anisotropic about the local magnetic field direction, potentially affecting the heating of the plasma as the turbulence is dissipated (e.g., Horbury et al. 2008, 2012; Wicks et al. 2010). In the case of SPC's radially aligned aperture, the derived temperature depends on the magnetic field direction with respect to the instrument's field of view (e.g., Huang et al. 2020). Therefore, it is important to investigate whether there is a significant correlation between the polytropic index values we derive and the magnetic field direction. We examine the $8 \mathrm{~s}$ subintervals of the same time period we examine in Section 4.5. For each $8 \mathrm{~s}$ subinterval, we calculate the mean $\cos \left(\theta_{\mathrm{Br}}\right)=B_{\mathrm{r}} / B$ and its standard deviation, where $\theta_{\mathrm{Br}}$ is the angle between the magnetic field vector and the radial direction. In the left panel of Figure 8, we show the 2D histogram of $\gamma$ and the mean $\cos \left(\theta_{\mathrm{Br}}\right)$. On the right and top 
2019 Apr. 2 00:00:00 UT - 2019 Apr. 4 00:00:00 UT
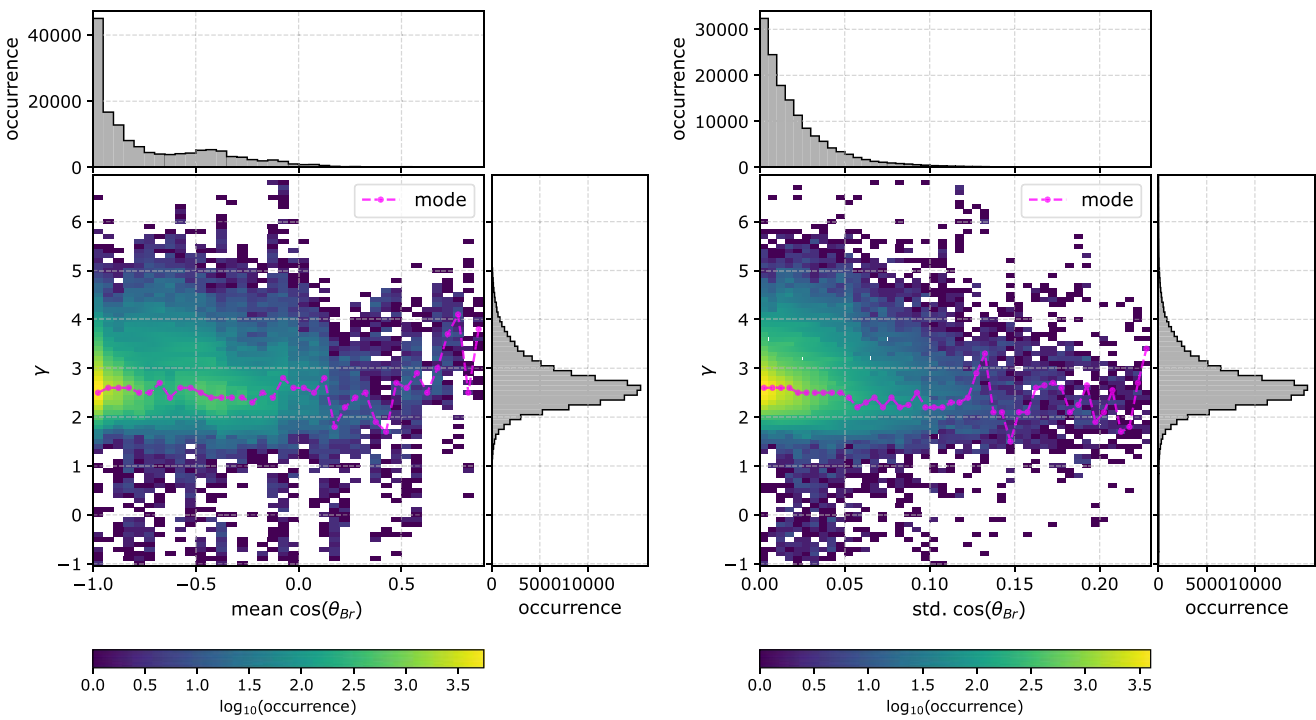

Figure 8. (Left) 2D histogram of $\gamma$ and the mean $\cos \left(\theta_{\mathrm{Br}}\right)$ and (right) $2 \mathrm{D}$ histogram of $\gamma$ and the standard deviation of $\cos \left(\theta_{\mathrm{Br}}\right)$ calculated for $8 \mathrm{~s}$ subintervals between 2019 April 2 00:00:00 UT and 2019 April 4 00:00:00 UT. On the top of each 2D histogram we show the 1D histograms of the mean cos $\left(\theta_{\mathrm{Br}}\right)$ and the standard deviation of $\cos \left(\theta_{\mathrm{Br}}\right)$, respectively, while on the right side we show the histogram of $\gamma$.

sides of the 2D histogram, we show the 1D histograms of the corresponding parameters. The magenta line shows the most frequent $\gamma$ in each $\cos \left(\theta_{\mathrm{Br}}\right)$ bin. The magnetic field is mainly radial $\left(\theta_{\mathrm{Br}} \sim 180^{\circ}\right)$, and the occurrence drops by a factor of about 4 as $\theta_{\mathrm{Br}}$ deviates by $\sim 30^{\circ}$ from the purely radial direction. There is no obvious correlation between $\gamma$ and $\cos \left(\theta_{\mathrm{Br}}\right)$. For all observed $B$-field directions, $\gamma \sim 2.7$, which is representative of the entire data set we analyze throughout this study. In the right panel of Figure 8, we show the 2D histogram of $\gamma$ and the standard deviation of $\cos \left(\theta_{\mathrm{Br}}\right)$. On the left and top sides of the 2D histogram we show the corresponding 1D histograms of the parameters. The standard deviation of $\cos \left(\theta_{\mathrm{Br}}\right)$ within the examined subintervals is less than 0.1 , which corresponds to less than $\sim 26^{\circ}$ from the radial direction. The magenta line shows the mode of $\gamma$ as a function of the standard deviation of $\cos \left(\theta_{\mathrm{Br}}\right)$. The most frequent $\gamma$ is greater than 2 in the entire range of observed standard deviations of $\cos \left(\theta_{\mathrm{Br}}\right)$, and there is no significant correlation between the two parameters. We note that standard deviations associated with random fluctuations would cause small random and systematic misestimations of the mathematical calculations of $\gamma$ values in the fitting (Nicolaou et al. 2019).

\section{Discussion}

\subsection{Large-scale Variations}

We investigate large-scale variations of the solar wind protons expanding in the heliosphere as observed by SPC in three time intervals at heliocentric distances between 0.17 and 1 au (Figure 1). In these three intervals, both the plasma density and temperature vary by two orders of magnitude. Besides their differences, which are possibly associated with the sampling of different wind streams, all 2D histograms in Figure 3 indicate that the large-scale variations of $n$ and $T$ follow a polytropic model with $\gamma \sim 5 / 3$, especially in the low density regime when $n<100 \mathrm{~cm}^{-3}$. A polytropic index with a value of $\sim 5 / 3$ corresponds to the near-adiabatic expansion model for plasma protons with three degrees of freedom $(f=3)$. In the higher density regime $\left(n>100 \mathrm{~cm}^{-3}\right)$, the slope of $\log _{10}(T)$ versus $\log _{10}(n)$ decreases, which possibly indicates heating of the protons or an increase of the effective degrees of freedom. In a similar approach, Totten et al. (1995) analyze Helios 1 observations and determine the polytropic relation of solar wind protons as functions of the heliocentric distance between 0.3 and 1 au. They derive an average $\gamma \sim 1.46$, which is below the adiabatic value for $f=3$, indicating that heating mechanisms act on the protons as they expand into the heliosphere. The authors also show that the value of $\gamma$ does not depend on the solar wind speed, which is often used as a criterion to distinguish between different origins of solar wind streams.

Hellinger et al. (2011) use Helios observations to study the plasma density, parallel and perpendicular temperature, speed, and magnetic field of protons in fast solar wind streams as functions of the radial distance within 0.3 and $1 \mathrm{au}$. The difference between their results and the predictions from the double adiabatic approach (e.g., Chew et al. 1956) leads the authors to argue for a mechanism, possibly of a kinetic nature, that cools the plasma in the direction parallel to the magnetic field and heats the plasma in the direction perpendicular to the magnetic field. The authors discuss a possible deceleration of secondary beams with respect to the core of the proton velocity distributions as explained by Marsch et al. (1982). Stansby et al. (2019) verify the corresponding energy transfer from the parallel to the perpendicular direction and show that alpha particles within the same heliocentric distance range exhibit a very similar behavior. The recent study by Huang et al. (2020) argues that PSP observes even more intense perpendicular heating and parallel cooling of the fast solar wind protons than observed by Helios.

We do not attempt to quantify a single relation between the plasma density and temperature that characterizes the entire data set within each of the three intervals we select. Our goal is to demonstrate the possible existence of a large-scale polytropic process characterizing the large-scale expansion of the solar wind protons in the heliosphere. Our analysis shows the existence of smaller-scale structures that deviate from the largescale behavior. 


\subsection{Profile of the Plasma Expansion}

The plasma density expansion within interval 1 exhibits features that clearly deviate from the $n \propto r^{-2}$ model for radial expansion with constant speed (Figure 4), which is widely used to describe the first-order density profile through the entire heliosphere (e.g., Richardson 2010). A change in the radial profile exponent could be associated with a nonradial expansion geometry or dynamic processes that decelerate or accelerate the plasma. However, our diagram in Figure 4 includes several stream crossings during the observations. Apparent deviations from the expected model may be due to nonuniform stream mixing.

In the bottom panel of Figure 4, we compare the observed temperature profile with three expansion models. Models with $1.5<\gamma<5 / 3$ capture the overall trend of the observations. The large-scale profile is consistent with a nearly adiabatic or subadiabatic expansion for plasma protons with three degrees of freedom. However, there are three regions with much steeper slopes, better described by $\gamma=2.7$, which is the typical value for the analysis of short-timescale subintervals. The three distinct regions we identify in Figure 4 are spread within the entire heliocentric distance range covered in interval 1.

As we show in Section 4.6 and discuss in Section 5.4 the temperature here represents the temperature tensor element along the aperture direction (radial), which is the typical magnetic field direction during the encounters due to the predominantly radial field geometry.

\subsection{Small-scale Fluctuations}

In the 2D histograms of Figure 3 and the radial expansion profiles in Figure 4, we distinguish subintervals that deviate from $\gamma \sim 5 / 3$. These are better described by a larger $\gamma$. The statistical analysis of short time subintervals verifies that smallscale fluctuations are frequently described by $\gamma \sim 2.7$ (see Figure 5). The $\gamma$ we derive is significantly greater than the values derived by previous analyses of short time intervals (selected streams) at larger heliocentric distances. Newbury et al. (1997) analyze solar wind protons within stream interaction regions at $\sim 0.74$ au observed by Pioneer Venus Orbiter. The authors analyze 73 stream interactions over time periods from 16 to $24 \mathrm{hr}$ and find intervals with $\gamma \sim 5 / 3$ and a few cases with $\gamma \sim 2$. The authors argue for a possible mechanism that occasionally acts on adiabatic plasma protons restricting their degrees of freedom resulting in a few occasions with $\gamma \sim 2$. If we consider an adiabatic process with $\gamma \sim 2$, then the process is restricted to $f=\frac{2}{\gamma-1} \sim 2$ degrees of freedom (see Section 1).

The analysis of solar wind proton streams at 1 au leads to a mean value of $\gamma \sim 1.8$ (e.g., Nicolaou et al. 2014a; Livadiotis 2018a, 2018b; Nicolaou \& Livadiotis 2019). These studies use proton measurements obtained with $\sim 1$ and $\sim 1.5$ minute resolution and calculate $\gamma$ within subintervals of $\sim 8$ minutes in length. Here, although we analyze subintervals of similar length, we determine a significantly greater $\gamma$. We note, however, that the majority of the analyzed subintervals in this study are obtained at heliocentric distances $<0.6$ au (see Figure 1). Therefore, our results, combined with the findings of the previous studies, suggest that the mechanisms that trigger fluctuations with $\gamma \sim 2.7$ are possibly less effective as the plasma propagates into the heliosphere. Such mechanisms could be associated with an energy exchange between proton

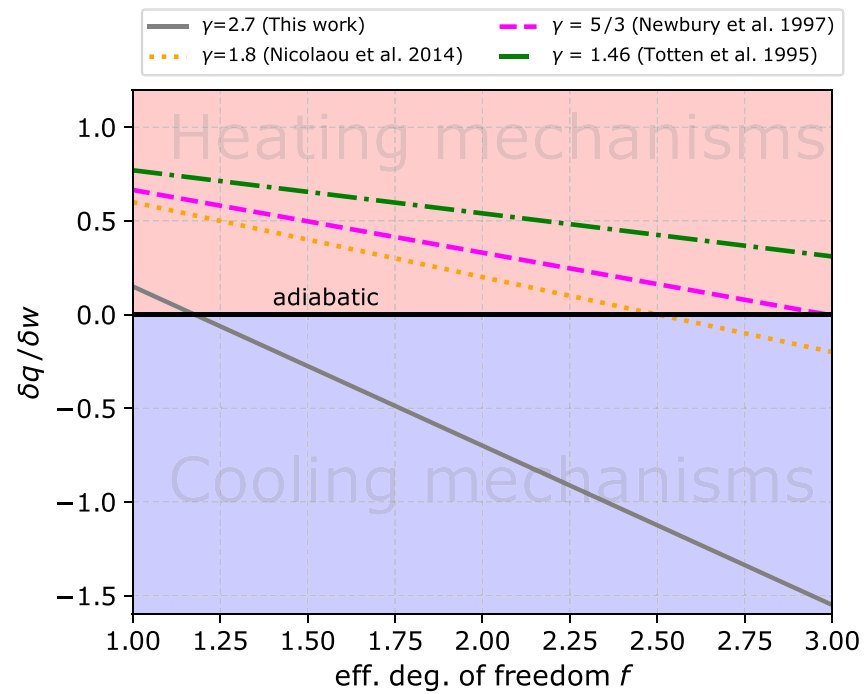

Figure 9. Diagram of $\delta q / \delta w$ as a function of the effective degrees of freedom $f=2\left(c_{\mathrm{p}} / c_{\mathrm{v}}-1\right)^{-1}$ for different $\gamma$ values. We explicitly show $\gamma=2.7$, which is the most frequent value we derive in this work, $\gamma=1.8$, which is the average proton polytropic index at 1 au according to Nicolaou et al. (2014a), $\gamma=5 / 3$, which characterizes the streams analyzed by Newbury et al. (1997), and $\gamma=1.46$ determined by Totten et al. (1995) for solar wind protons between 0.3 and $1 \mathrm{au}$. The red and blue shadows indicate a set of parameter values consistent with mechanisms supplying and retaining heat from an expanding plasma, respectively.

particles and fluctuations and/or the restriction of the proton effective degrees of freedom by introducing temperature anisotropies. It is also possible that, at larger heliocentric distance, different streams with $\gamma \sim 2.7$ get well-mixed, resulting in streams with an overall $\gamma \sim 5 / 3$. The identification of specific mechanisms responsible for this result requires further analyses of the velocity distribution functions of all plasma species and the magnetic fields.

In order to quantify the possible heating or cooling of the plasma, we express $\gamma$ in terms of the particle effective degrees of freedom $f=2\left(c_{\mathrm{p}} / c_{\mathrm{v}}-1\right)^{-1}$. We express the ratio of the energy supplied to the system as heat and the energy supplied as work as $\delta q / \delta w$. Starting from the first law of thermodynamics and following the algebra described by Livadiotis (2019), we get:

$$
\gamma=\frac{2}{f}\left(1-\frac{\delta q}{\delta w}\right)+1
$$

In Figure 9, we show $\delta q / \delta w$ as a function of $f$ for different $\gamma$ values determined for solar wind protons in different studies. The red regions indicate sets of values consistent with mechanisms supplying heat to the expanding plasma $(\delta q /$ $\delta w>0)$, while the blue regions correspond to values consistent with mechanisms retaining heat from the expanding plasma $(\delta q / \delta w<0)$. Therefore, for a given $\gamma$, this diagram enables us to determine the range of possible effective degrees of freedom $f$ that are associated with heating and/or cooling mechanisms According to the diagrams, $\gamma \sim 2.7$ characterizes an adiabatic plasma only if $f \sim 1$. Our results are largely consistent with the kinetic description of plasma ions interacting with slow waves, where ions behave as if they are a one-dimensional $(f=1)$ adiabatic fluid $(\delta q=0)$ with temperature variations confined along the magnetic field (e.g., Gary 1993; Verscharen et al. 2017). On the other hand, in a case with $f>1$, our study 
implies the existence of mechanisms that cool the plasma protons, at least in the observed direction of phase space density.

\subsection{Polytropic Behavior and Plasma Properties}

At distances closer to the Sun than previously studied, the polytropic index does not depend on the plasma speed. The polytropic index is largely independent on speed at larger heliocentric distances as well (Totten et al. 1995; Livadiotis 2018b; Nicolaou \& Livadiotis 2019), supporting the argument that plasma of different solar source regions does not exhibit different polytropic behavior. Characteristically, the annual average of $\gamma$ characterizing the solar wind protons at 1 au over the last two solar cycles does not depend on the solar wind speed, and its all-year mean value is $\gamma=1.86 \pm 0.09$ (Livadiotis 2018b).

On the other hand, we analyze a short time interval in which $\gamma$ reduces with increasing plasma $\beta$. According to our discussion in 5.2 and the diagrams in Figure 9, the observed negative correlation between $\gamma$ and $\beta$ could be due to an increase of $f$ or/and an increase in $\delta q / \delta w$ with increasing $\beta$. When strong magnetic fields dominate the particle thermal motions (low $\beta$ ), the thermodynamic processes are confined along the direction of the magnetic field and, thus, the effective degrees of freedom are reduced, or/and there is a mechanism that effectively absorbs energy from the plasma protons.

Our examination of $\gamma$ as a function of the magnetic field direction relative to the instrument's aperture and its fluctuations does not show any significant systematic trend, eliminating the possibility of artifacts related to the 1D measurement of the plasma temperature. Our histograms in Figure 8 show that, for most of the time, the magnetic field is radial and thus the measured temperature values correspond mostly to the temperature parallel to the magnetic field.

\section{Conclusions}

Our study examines the large-scale and the small-scale variations of the plasma proton density and temperature as observed by the PSP SPC instrument at heliocentric distances between $\sim 0.17$ and $\sim 0.8$ au. We conclude that:

1. Large-scale plasma proton variations due to solar wind expansion in the heliosphere tend to follow a polytropic model with $1.5<\gamma<5 / 3$.

2. The small-scale fluctuations associated with turbulent compressions (or other local variations) follow a polytropic relation with $\gamma \sim 2.7$, indicating a process with significant cooling or/and restriction of the particle effective degrees of freedom.

3. Our comparison with studies at 1 au suggests that the mechanisms that trigger fluctuations with $\gamma \sim 2.7$ occur predominantly near the Sun and/or small-scale fluctuations are possibly blended in streams with $\gamma \sim 5 / 3$ as the plasma propagates into the heliosphere.

4. The polytropic index of the small-scale fluctuations does not depend on the plasma flow speed or the direction of the magnetic field.

5. We analyze a case in which the polytropic index exhibits a negative correlation with the plasma $\beta$. It would be worthwhile to explore this dependence further in future statistical studies of 3D particle distributions. These studies should investigate whether plasma with low $\beta$ exhibits fewer effective degrees of freedom or whether yet to be identified mechanisms remove thermal energy from the protons in the inner heliosphere.

6. Future coherent studies of the full temperature tensor of all plasma species (protons, electrons, and heavy ions), the detailed characterization of their 3D distributions, and the magnetic field will extend our knowledge of the plasma heating mechanisms. We highlight the importance of future 3D analyses by the SPAN electrostatic-analyzer instrument on PSP and the Solar Wind Analyser instrument suite (Owen et al. 2020) on board Solar Orbiter.

G.N., R.T.W, and D.V. are supported by the STFC Consolidated grant to UCL/MSSL, ST/S000240/1. G.L. is supported by NASA's project 80NSSC19K0079. D.V. is supported by STFC Ernest Rutherford Fellowship ST/ P003826/1. This research made use of HelioPy, a community-developed Python package for space physics (Stansby et al. 2020). We use the publicly available SWEAP and FIELDS data found at https://spdf.gsfc.nasa.gov/pub/data/ psp. We acknowledge the NASA Parker Solar Probe Mission, SWEAP team led by Justin Kasper and Fields team led by Stuart Bale for use of data.

\section{ORCID iDs}

George Livadiotis (iD https://orcid.org/0000-0002-7655-6019 Robert T. Wicks (iD https://orcid.org/0000-0002-0622-5302 Daniel Verscharen (ib https://orcid.org/0000-0002-0497-1096 Bennett A. Maruca (iD https://orcid.org/0000-0002-2229-5618

\section{References}

Arridge, C. S., McAndrews, H. J., Jackman, C. M., et al. 2009, P\&SS, 57, 2032 Bale, S. D., Goetz, K., Harvey, P. R., et al. 2016, SSRv, 204, 49

Bavassano, B., Bruno, R., \& Rosenbauer, H. 1996, AnGeo, 14, 510

Borovsky, J. E. 2016, JGRA, 121, 5055

Bruno, R., \& Carbone, V. 2013, LRSP, 10, 2

Chandrasekhar, S. 1967, An Introduction to the Study of Stellar Structure (New York: Dover Publications)

Chen, C. H. K., Leung, L., Boldyrev, S., et al. 2014, GeoRL, 41, 8081

Chew, G. F., Goldberger, M. L., \& Low, F. E. 1956, RSPSA, 236, 112

Dialynas, K., Roussos, E., Regoli, L., et al. 2018, JGRA, 123, 8066

Elliott, H. A., McComas, D. J., Zirnstein, E. J., et al. 2019, ApJ, 885, 156

Franci, L., Landi, S., Matteini, L., et al. 2016, ApJ, 833, 91

Gary, S. P. 1993, Theory of Space Plasma Microinstabilities (New York: Cambridge Univ. Press)

Geiss, J., Gloeckler, G., \& Von Steiger, R. 1995, SSRv, 72, 49

Hellinger, P., Matteini, L., Štverák, Š., Trávníéek, P. M., \& Marsch, E. 2011, JGRA, 116, A09105

Horbury, T. S., Forman, M. A., \& Oughton, S. 2008, PhRvL, 101, 175005

Horbury, T. S., Wicks, R. T., \& Chen, C. H. K. 2012, SSRv, 172, 325

Huang, J., Kasper, J. C., Vech, D., et al. 2020, ApJS, 246, 70

Kartalev, M., Dryer, M., Grigorov, K., et al. 2006, JGR, 111, A10107

Kasper, J. C., Abiad, R., Austin, G., et al. 2016, SSRv, 204, 131

Kuhn, S., Kamran, M., Jelić, N., et al. 2010, in AIP Conf. Proc. 1306, New Frontiers in Advanced Plasma Physics (Melville, NY: AIP), 216

Livadiotis, G. 2015, ApJ, 809, 111

Livadiotis, G. 2016, ApJs, 223, 13

Livadiotis, G. 2018a, JGRA, 123, 1050

Livadiotis, G. 2018b, Entr, 20, 799

Livadiotis, G. 2019, Entr, 21, 1041

Livadiotis, G., \& Desai, M. I. 2016, ApJ, 829, 88

Livadiotis, G., \& McComas, D. J. 2013, JGRA, 118, 2863

Livadiotis, G., McComas, D. J., Dayeh, M. A., et al. 2011, ApJ, 734, 1

Livadiotis, G., McComas, D. J., Schwadron, N. A., et al. 2013, ApJ, 762, 134 Marsch, E. 2006, LRSP, 3, 1

Marsch, E., Mühlhäuser, K. H., Rosenbauer, H., et al. 1983, JGR, 88, 2982

Marsch, E., Mühlhäuser, K. H., Schwenn, R., et al. 1982, JGR, 87, 52 
McComas, D. J., Elliott, H. A., Schwadron, N. A., et al. 2003, GeoRL, 30, 1517

Newbury, J. A., Russel, C. T., \& Lindsay, G. M. 1997, GeoRL, 24, 1431

Nicolaou, G., \& Livadiotis, G. 2017, ApJ, 838, 7

Nicolaou, G., \& Livadiotis, G. 2019, ApJ, 884, 52

Nicolaou, G., Livadiotis, G., \& Moussas, X. D. 2014a, SoPh, 289, 1371

Nicolaou, G., Livadiotis, G., \& Wicks, R. T. 2019, Entr, 21, 997

Nicolaou, G., McComas, D. J., Bagenal, F., \& Elliott, H. A. 2014b, JGRA, 119,3463

Osherovich, V. A., Farrugia, C. J., Burlaga, L. F., et al. 1993, JGR, 98, 15331

Owen, C. J., Bruno, R., Livi, S., et al. 2020, A\&A, in press

Pang, X., Cao, J., Liu, W., et al. 2015, JGRA, 120, 4736

Park, J.-S., Shue, J.-H., Nariyuki, Y., et al. 2019, JGRA, 124, 1866

Parker, E. N. 1961, ApJ, 134, 20

Parker, E. N. 1963, Interplanetary Dynamical Processes (New York: WileyInterscience)

Perrone, D., Stansby, D., Horbury, T. S., et al. 2019, MNRAS, 483, 3730
Richardson, J. D. 2010, in Heliophysical Processes, Astrophysics and Space Science Proc., ed. N. Gopalswamy, S. Hasan, \& A. Ambastha (Berlin: Springer), 83

Scherer, K., Fichtner, H., Fahr, H. J., et al. 2016, ApJ, 833, 38

Spreiter, J. R., \& Stahara, S. S. 1994, AdSpR, 14, 5

Stansby, D., Perrone, D., Matteini, L., et al. 2019, A\&A, 623, L2

Stansby, D., Rai, Y., \& Argall, M. 2020, heliopython/heliopy: HelioPy v0.10.1, Zenodo, doi:10.5281/zenodo.3739114

Totten, T. L., Freeman, J. W., \& Arya, S. 1995, JGR, 100, 13

Verscharen, D., Chandran, B. D. G., Klein, K. G., et al. 2016, ApJ, 831, 128

Verscharen, D., Chen, C. H. K., \& Wicks, R. T. 2017, ApJ, 840, 106

Verscharen, D., Klein, K. G., \& Maruca, B. A. 2019, LRSP, 16, 5

Wicks, R. T., Horbury, T. S., Chen, C. H. K., \& Schekochihin, A. A. 2010, MNRAS, 407, L31

Woodham, L. D., Wicks, R. T., Verscharen, D., et al. 2019, ApJL, 884, L53

Wu, H., Verscharen, D., Wicks, R. T., et al. 2019, ApJ, 870, 106 